\title{
Correlation analysis between femoral trochlear dysplasia and anterior cruciate ligament injury based on CT measurement
}

\author{
Mengyu Chen ${ }^{1 \#}$, Le Qin ${ }^{2 \#}$, Mei Li $^{1}$, Ji Shen $^{3}$ \\ ${ }^{1}$ Department of Radiology, Shanghai Jiao Tong University Affiliated Sixth People's Hospital, Shanghai 200233, China; ${ }^{2}$ Department of Radiology, \\ Shanghai Jiao Tong University Medical School Affiliated Ruijin Hospital, Shanghai 200025, China; ${ }^{3}$ Department of Orthopaedics, Shanghai Jiao \\ Tong University Affiliated Sixth People's Hospital, Shanghai 200233, China \\ \#These authors contributed equally to the work.
}

Correspondence to: Mei Li. Department of Radiology, Shanghai Jiao Tong University Affiliated Sixth People's Hospital, Shanghai 200233, China. Email: baoxinyi51@163.com.

\begin{abstract}
Background: Anterior cruciate ligament (ACL) injury is the most common knee ligament injury. Femoral trochlear dysplasia (FTD) is an anatomic deformity characterized by a shallow trochlear angle and depth. There is limited research on the correlation between ACL injury and trochlear dysplasia.

Methods: A total of 423 patients with an ACL injury who underwent ACL reconstruction and plain computed tomography (CT) follow-up scan one week after the operation in our hospital between January 2017 and March 2019 were enrolled in this study. A total of 352 patients (369 knees) without ACL injury served as a control group. The sulcus angle, trochlear facet asymmetry, trochlear depth, and lateral trochlear inclination were measured at $3 \mathrm{~cm}$ above the tibiofemoral joint. The notch width index (NWI) was measured on the CT axis, and the FTD was divided into four types according to the Dejour classification.

Results: The prevalence of FTD was significantly higher in the ACL injury group than in the control group $\left(30.7 \%\right.$ vs. $\left.14.4 \%, \chi^{2}=30.834, \mathrm{P}<0.001\right)$. The trochlear sulcus angle was higher, while the trochlear facet asymmetry and lateral trochlear inclination were significantly lower in the ACL injury group than in the control group $(\mathrm{P}<0.01)$. The NWI was 0.232 in the ACL injury group and 0.245 in the control group $(\mathrm{P}<0.01)$. In the ACL injury group, the NWI of patients with FTD was slightly smaller than that of the patients without FTD $(\mathrm{P}<0.01)$.
\end{abstract}

Conclusions: Our findings indicate that FTD might be a significant risk factor for ACL injury. Whether FTD is related to ACL injury caused by smaller intercondylar notch needs further study.

Keywords: Femoral trochlear dysplasia (FTD); anterior cruciate ligament injury (ACL injury); intercondylar notch

Submitted Sep 10, 2019. Accepted for publication Mar 16, 2020.

doi: 10.21037/qims.2020.03.15

View this article at: http://dx.doi.org/10.21037/qims.2020.03.15

\section{Introduction}

Anterior cruciate ligament (ACL) injury is the most common knee ligament injury. Previous studies have shown that narrow intercondylar fossa, steeper posterior tibial slope, and small ACL volume are associated with ACL injury (1-3). The trochlear groove forms the femoral articular surface of the patellofemoral joint. The depth and anatomical shape of the trochlear groove affect patellar stability during knee flexion and extension (4). Femoral trochlear dysplasia (FTD) is a congenital morphological abnormality characterized by a shallow trochlear angle and depth, which could be assessed by measuring the sulcus angle, trochlear facet asymmetry, trochlear depth, and lateral trochlear inclination on axial- 


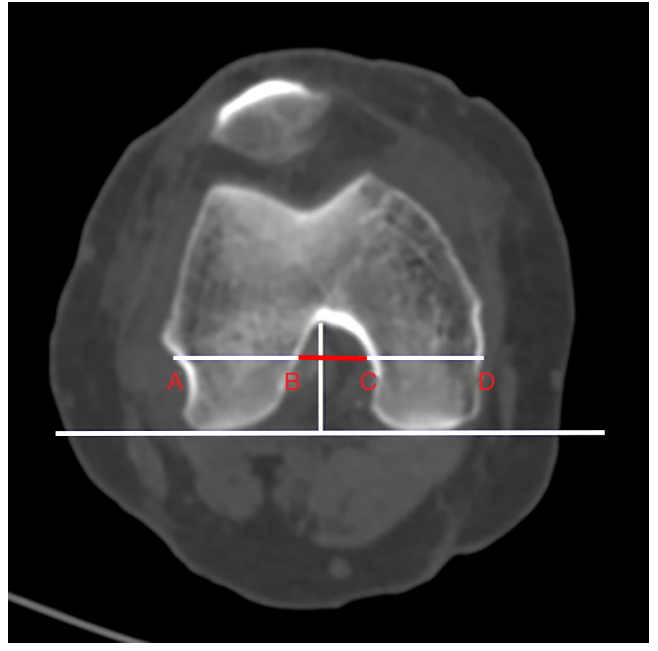

Figure 1 Measurement of the femoral notch width index (NWI) on an axial image. The notch depth is perpendicular to a line connecting the ventral articular surfaces of the medial and lateral femoral condyle. The notch width and condylar width are parallel to the line at $2 / 3$ of the notch depth. $A B$ : notch width; $C D$ : condylar width. $\mathrm{NWI}=\mathrm{AB} / \mathrm{CD}$.

view computed tomography (CT) or magnetic resonance imaging (MRI) (5-7).

At present, there is limited research on the correlation between ACL injury and FTD. Ntagiopoulos et al. retrospectively analyzed the incidence of FTD in 299 knees with ACL injury (8). They found that the incidence of FTD in patients with ACL injury (14.7\%) was higher than that recorded in control groups in other studies (2-3\%), but there was no significant correlation between FTD and ACL injury. Botchu et al. determined a significant correlation between type A trochlear dysplasia and ACL injury while investigating 95 patients with anterior knee pain (9), while Van Haver et al. reported that femurs with trochlear dysplasia were also characterized by a smaller intercondylar notch $(10,11)$.

The femoral trochlea is formed by the central sulcus and bilateral cartilage surfaces, and the trochlear notch extends distally to the intercondylar notch; we thus hypothesized that FTD might cause abnormal anatomy of the intercondylar notch, which might be a predisposition to an ACL injury. In this study, we investigated the association between FTD and ACL injury by comparing the postoperative CT data of patients with ACL injury against the CT data of patients without ACL injury. We hypothesized that (I) the intercondylar notch of patients with FTD is smaller than that of patients with normal trochlea, and (II) patients with FTD are more prone to an ACL injury.

\section{Methods}

\section{Research object}

The population in this retrospective observational study consisted of 423 patients with an ACL injury. They underwent ACL reconstruction and a follow-up plain CT scan one week after the operation in our hospital from January 2017 to March 2019. A total of 352 patients (369 knees) who had a history of a knee injury but no evidence of ACL injury on CT scans, MRI scans, or physical examination, served as the control group. As Wang et al. (12) suggest that only excessive accumulation of fluid in the joint is pathological; patients with a slight degree knee effusion were included in the control group. Patients with a history of knee surgery, knee fracture, osteoarthritis, loose body, or lower-extremity force line abnormality, and those older than 35 years or younger than 18 years were excluded.

\section{CT imaging and analysis}

All patients were scanned by a 64-slice multidetector CT scanner (Lightspeed VCT 64, GE, Milwaukee, WI, USA) in the supine position with the knee extended. The scanning parameters were as follows: tube voltage, $120 \mathrm{KV}$; tube current, $350 \mathrm{~mA}$; slice thickness, $5 \mathrm{~mm}$; reconstructive thickness, $0.625 \mathrm{~mm}$. According to Pfirrmann's method (13), the sulcus angle, trochlear facet asymmetry, trochlear depth, and lateral trochlear inclination were measured at $3 \mathrm{~cm}$ above the tibiofemoral joint. The notch width index (NWI) was used to evaluate intercondylar notch stenosis. NWI was calculated as the intercondylar notch width divided by the femoral condylar width measured on the CT axis, according to Stein's method (14) (Figure 1). According to the Dejour classification, FTD was classified into type A (shallow trochlear sulcus), B (flat or convex trochlea), C (hypoplastic medial facet), or D (cliff-shaped trochlea). Two musculoskeletal radiologists performed the measurements. The prevalence of FTD and the measured parameters were compared between the two groups.

\section{Statistical analysis}

Statistical analysis was performed by the Statistical Product 
and Service Solutions (SPSS) software package, version 22.0 (IBM Corp., Armonk, NY, USA). The chi-square test was used to analyze qualitative parameters (prevalence of trochlear dysplasia), and the independent $t$-test was used to analyze quantitative parameters (sulcus angle, trochlear facet asymmetry, trochlear depth, lateral trochlear inclination, and NWI). All quantitative parameters conformed to the normal distribution. The level of statistical significance was set to $\mathrm{P}<0.05$.

\section{Results}

Of the 423 patients (334 males and 89 females) with an ACL injury, 203 had a left ACL complete tear, and 220 had a right ACL complete tear. Their age ranged from 18 to 35 years, with an average of 26.9 years. There were 297 patients (314 knees) without evidence of ACL injury on clinical symptoms, CT scans, or physical examination (anterior drawer test and Lachman test). The other87patients who were suspected of ACL injury after CT scanning and physical examination underwent MRI scanning, the results of which indicated that 32 of these cases had an ACL injury, and 55 cases had a normal ACL. Therefore, 352 patients (369 knees), including one patient

Table 1 Comparison of the number of Dejour types between the ACL injury group and control group

\begin{tabular}{lcc}
\hline Dejour type & $\begin{array}{c}\text { The ACL injury group } \\
(\mathrm{n}=423)\end{array}$ & $\begin{array}{c}\text { The control group } \\
(\mathrm{n}=369)\end{array}$ \\
\hline Type A & 71 & 26 \\
Type B & 39 & 17 \\
Type C & 9 & 4 \\
Type D & 11 & 6 \\
Normal & 293 & 316 \\
\hline
\end{tabular}

$\mathrm{ACL}$, anterior cruciate ligament. with a partial medial collateral ligament injury and five patients with meniscus injury on MRI, constituted the control group. The control group consisted of 196 left knees and 173 right knees. The mean age in the control group was 27.6 years (range, 18-35 years). There were 193 males and 159 females. One hundred and thirty patients in the ACL injury group and 53 patients in the control group had FTD. The prevalence of FTD was significantly higher in the ACL injury group than in the control group $(30.7 \% v s$. $\left.14.4 \%, \chi^{2}=30.834, \mathrm{P}<0.001\right)$. Table 1 shows the distribution of FTD types in the two groups. The trochlear sulcus angle was higher, while the trochlear facet asymmetry and lateral trochlear inclination were significantly lower in the ACL injury group than in the control group $(\mathrm{P}<0.01)$. The trochlear depth was lower in the ACL injury group than in the control group, but there was no significant difference $(\mathrm{P}=0.140$ ) (Table 2). The NWI was 0.232 in the ACL injury group and 0.245 in the control group $(\mathrm{P}<0.01)$. Also, in the ACL injury group, the average NWI of patients with FTD was 0.228 , and that of patients with normal trochlea was 0.235 , with this difference being significant $(\mathrm{P}<0.01)$. In the control group, the average NWI of patients with FTD was 0.240 , and that of patients with normal trochlea was 0.246 , without a significant difference $(\mathrm{P}=0.073)$.

\section{Discussion}

The most crucial finding in this study was that the prevalence of FTD was higher in patients with ACL injury than in those of the control group, indicating that FTD might be a risk factor for ACL injury. Also, we found that patients with ACL injury had a narrower intercondylar notch than those in the control group. The NWI in patients with FTD was slightly smaller than that in patients with normal trochlea in the ACL injury group.

In individuals with FTD, the proximal trochlear joint surface is flattened, and the distal concavity is less distinct. In more severe cases, the trochlear surface might even

Table 2 Comparison of anatomical parameters between the ACL injury group and the control group

\begin{tabular}{lccc}
\hline FTD anatomical parameters & ACL injury group $(\mathrm{n}=423)$ & Control group $(\mathrm{n}=369)$ & $\mathrm{P}$ value \\
\hline Sulcus angle & $153.42 \pm 8.61$ & $149.18 \pm 8.18$ & 0.000 \\
Trochlear depth & $4.15 \pm 1.36$ & $4.29 \pm 1.23$ & 0.140 \\
Trochlear facet asymmetry & $55.03 \% \pm 14.51 \%$ & $61.30 \% \pm 14.16 \%$ & 0.000 \\
Lateral trochlear inclination & $18.36 \pm 4.72$ & $19.53 \pm 4.79$ & 0.001 \\
\hline
\end{tabular}

$\mathrm{ACL}$, anterior cruciate ligament; FTD, femoral trochlear dysplasia. 
become convex with increasing hypoplasia of the medial joint surface (7). Normal trochlear anatomy is essential for the biomechanics of knee extension. ACL reconstruction does not affect the trochlear morphology or parameters. CT provides a more accurate bony morphology for the measurement of FTD parameters and better information on the tunnel and intramedullary nail location. Also, it is more time-saving and convenient than MRI, and the data are highly reproducible. Therefore, we selected patients who underwent ACL reconstruction and follow-up plain CT 1 week after the operation into our study group. However, it produces ionizing radiation with the respective risks to patients, particularly to fertile women. We suggest that MRI should be performed preoperatively owing to its high accuracy in diagnosing ACL injury. CT scan should be performed after informing patients of the risk of radiation for postoperative evaluation.

The ACL, which starts from the medial wall of the lateral femoral condyle in the intercondylar notch, is one of the major stabilizing ligaments of the knee joint. Its function is to maintain the static and dynamic stability of the knee with other structures and to restrict tibial rotation and forward movement. An ACL injury causes knee instability, leading to many chronic knee diseases, such as meniscus and cartilage injury, along with osteoarthritis. Different mechanisms cause an ACL injury. Intrinsic anatomic variables, such as a narrow intercondylar notch or a steeper posterior tibial slope, are known to be associated with an ACL injury. Narrowing of the intercondylar notch is considered to be a risk factor for ACL injury, as first described by Palmer (15). After that, a large number of studies have suggested that intercondylar notch stenosis is a risk factor for ACL injury and that the NWI of patients with an ACL injury is smaller than that of normal people (16-19). As for the NWI, it is calculated by dividing the intercondylar notch width by the femoral condylar width. According to Souryal, NWI can eliminate the interference of age, sex, height, weight, and other factors, while reducing errors, standardizing measurement results, and reflecting the degree of intercondylar stenosis accurately (19).

At present, few clinical studies are reporting on the correlation between ACL injury and FTD. Botchu et al. found a significant correlation between type A trochlear dysplasia and ACL injury, but they did not study the anatomical parameters reflecting the trochlear shape, such as the sulcus angle or trochlear depth (9). In our study, using the measurement of the trochlear anatomical parameters, we found that the trochlear sulcus angle was higher, while the trochlear facet asymmetry and lateral trochlear inclination were lower in patients with ACL injury than in those of the control group. Moreover, the prevalence of FTD was significantly higher in patients with an ACL injury. Hence, we speculate that FTD is a risk factor for an ACL tear. Van Haver's analysis revealed that the femur with trochlear dysplasia had a $10 \%$ smaller intercondylar notch compared to that of a normal femur $(10,11)$. Therefore, we speculate that FTD might cause abnormal anatomy of the intercondylar notch, which might be a predisposition to an ACL injury.

Muneta proposed two hypotheses when studying the relationship between ACL volume and intercondylar fossa size (20). The first stated that a narrow notch would have a correspondingly smaller ACL and that a smaller ACL would be less strong and more likely to rupture. The second stated that a narrow notch surrounding a normal size ACL would provide insufficient space for the ligament to function normally. The ACL does not impact the femoral intercondylar notch, and it has no friction with its lateral wall during knee flexion and extension in normal anatomical structure. A stenotic intercondylar notch causes ACL impingement, from either the medial aspect of the lateral femoral condyle or the intercondylar roof of the notch. It might result in an increased concentration of stresses on the midsubstance of the ACL during knee extension when the ACL contacts the top of the notch, and an increased force on the ligament when it is stretched across the lateral femoral condyle inflection $(21,22)$. Comerford et al. demonstrated that ACL impingement caused by an arrow intercondylar notch might reduce the structural integrity of the ligament (23).

Moreover, an altered ACL composition might predispose the ligament to increased laxity, which would lead to knee instability and would aggravate the narrow intercondylar notch. In our study, the NWI in patients with FTD was slightly lower than that in patients with normal trochlea in both groups. There was a significant difference with the ACL injury group. Therefore, it is necessary to measure parameters such as intercondylar height and notch shape index to verify whether the intercondylar notch of patients with FTD is smaller than that of patients with normal trochlea.

We believe that FTD changes the knee biomechanics, leading to increased ACL load and injury risk. Liu and Biedert found a smaller lateral posterior condyle and a larger the medial posterior condyle in patients with FTD when compared with those with normal trochlea $(24,25)$. 
During axial torsion, the distal femur tends to internally rotate due to the smaller lateral posterior and larger medial posterior condyle. Bretin found that internal rotation would result in valgus deviation of the mechanical axis and a shift in the center of force towards the lateral condyle, which would increase the stress on the ACL (26).

There are several limitations to the present study. First, this is a single-center retrospective study, resulting in a small number of recruited patients. Second, the trauma severity and mechanism might have affected the analysis of the association between ACL injury and FTD. In order to reduce the error, we need to obtain all relevant information for further analysis, including the injury mechanism and severity. Finally, it is necessary to measure parameters such as the intercondylar height and notch shape index to verify further whether the intercondylar notch of patients with FTD is smaller than that of patients with normal trochlea.

In conclusion, the prevalence of FTD was higher in patients with an ACL injury. The anatomical differences between patients with and without ACL injury indicated that FTD might be a risk factor for ACL injury. FTD might lead to a small intercondylar notch and change in the knee biomechanics, resulting in increased ACL injury risk. Therefore, we suggest that young people, particularly athletes, should be evaluated for FTD whenever possible to avoid ACL injury.

\section{Acknowledgments}

Funding: This study sponsored by the interdisciplinary Program of Shanghai Jiao Tong University (project number: YG2017MS20) and grant 2017ZZ02005 from the Shanghai Key Discipline of Medical Imaging.

\section{Footnote}

Conflicts of Interest: All authors have completed the ICMJE uniform disclosure form (available at http://dx.doi. org/10.21037/qims.2020.03.15). The authors have no conflicts of interest to declare.

Ethical Statement: This retrospective study was approved by the Institutional Ethics Committee of our hospital, which waived the requirement for written informed consent.

Open Access Statement: This is an Open Access article distributed in accordance with the Creative Commons Attribution-NonCommercial-NoDerivs 4.0 International
License (CC BY-NC-ND 4.0), which permits the noncommercial replication and distribution of the article with the strict proviso that no changes or edits are made and the original work is properly cited (including links to both the formal publication through the relevant DOI and the license). See: https://creativecommons.org/licenses/by-nc$\mathrm{nd} / 4.0 /$.

\section{References}

1. Chaudhari AMW, Zelman EA, Flanigan DC, Kaeding CC, Nagaraja HN. Anterior Cruciate Ligament-Injured Subjects Have Smaller Anterior Cruciate Ligaments than Matched Controls. Am J Sports Med 2009;37:1282-7.

2. Hoteya K, Kato Y, Motojima S, Ingham SJ, Horaguchi T, Saito A. Association between intercondylar notch narrowing and bilateral anterior cruciate ligament injuries in athletes. Arch Orthop Trauma Surg 2011;131:371-6.

3. Simon RA, Everhart JS, Nagaraja HN, Chaudhari AM. A case-control study of anterior cruciate ligament volume, tibial plateau slopes and intercondylar notch dimensions in ACL-injured knees. J Biomech 2010;43:1702-7.

4. Greiwe RM, Saifi C, Ahmad CS, Gardner TR. Anatomy and Biomechanics of Patellar Instability. Oper Techn Sport Med 2010;18:62-7.

5. Lewallen LW, McIntosh AL, Dahm DL. Predictors of Recurrent Instability after Acute Patellofemoral Dislocation in Pediatric and Adolescent Patients. Am J Sports Med 2013;41:575-81.

6. Fithian DC, Paxton EW, Stone ML, Silva P, Davis DK, Elias DA, White LM. Epidemiology and Natural History of Acute Patellar Dislocation. Am J Sports Med 2004;32:1114-21.

7. Diederichs G, Issever AS, Scheffler S. MR Imaging of Patellar Instability: Injury Patterns and Assessment of Risk Factors. RadioGraphics 2010;30:961-81.

8. Ntagiopoulos PG, Bonin N, Sonnery-Cottet B, Badet R, Dejour D. The incidence of trochlear dysplasia in anterior cruciate ligament tears. Int Orthop 2014;38:1269-75.

9. Botchu R, Obaid H, Rennie WJ. Correlation between Trochlear Dysplasia and Anterior Cruciate Ligament Injury. J Orthop Surg (Hong Kong) 2013;21:185-8.

10. Van Haver A, De Roo K, De Beule M, Van Cauter S, Audenaert E, Claessens T, Verdonk P. Semi-automated landmark-based 3D analysis reveals new morphometric characteristics in the trochlear dysplastic femur. Knee Surg Sports Traumatol Arthrosc 2014;22:2698-708.

11. Van Haver A, Mahieu P, Claessens T, Li H, Pattyn C, 
Verdonk P, Audenaert EA. A statistical shape model of trochlear dysplasia of the knee. Knee 2014;21:518-23.

12. Wang L, Xiang X, Tang Y, Yang Y, Qiu L. Sonographic appearance of fluid in peripheral joints and bursaeof healthy asymptomatic Chinese population. Quant Imaging Med Surg 2018;8:781-7.

13. Pfirrmann CW, Zanetti M, Romero J, Hodler J. Femoral trochlear dysplasia: MR findings. Radiology 2000;216:858-64.

14. Stein V, Li L, Guermazi A, Zhang Y, Kent Kwoh C, Eaton CB, Hunter DJ, OAI Investigators. The relation of femoral notch stenosis to ACL tears in persons with knee osteoarthritis. Osteoarthritis Cartilage 2010;18:192-9.

15. Palmer I. On the Injuries to the Ligaments of the Knee Joint: A Clinical Study. Clin Orthop Relat Res 2007;454:17-22; discussion 14.

16. Domzalski M, Grzelak P, Gabos P. Risk factors for Anterior Cruciate Ligament injury in skeletally immature patients: analysis of intercondylar notch width using Magnetic Resonance Imaging. Int Orthop 2010;34:703-7.

17. Park JS, Nam DC, Kim DH, Kim HK, Hwang SC. Measurement of Knee Morphometrics Using MRI: A Comparative Study between ACL-Injured and NonInjured Knees. Knee Surg Relat Res2012;24:180-5.

18. Keays SL, Keays R, Newcombe PA. Femoral intercondylar notch width size: a comparison between siblings with and without anterior cruciate ligament injuries. Knee Surg Sports Traumatol Arthrosc 2016;24;672-9.

19. Souryal TO, Freeman TR. Intercondylar notch size and anterior cruciate ligament injuries in athletes. A

Cite this article as: Chen $M$, Qin L, Li M, Shen J. Correlation analysis between femoral trochlear dysplasia and anterior cruciate ligament injury based on CT measurement. Quant Imaging Med Surg 2020;10(4):847-852. doi: 10.21037/ qims.2020.03.15 prospective study. Am J Sports Med 1993;21:535-9.

20. Muneta T, Takakuda K, Yamamoto H. Intercondylar Notch Width and its Relation to the Configuration and Cross-Sectional Area of the Anterior Cruciate Ligament. Am J Sports Med 1997;25:69-72.

21. Norwood LA, Cross MJ. The intercondylar shelf and the anterior cruciate ligament. Am J Sports Med 1977;5:171-6.

22. Swami VG, Mabee M, Hui C, Jaremko JL. ThreeDimensional Intercondylar Notch Volumes in a Skeletally Immature Pediatric Population: A Magnetic Resonance Imaging-Based Anatomic Comparison of Knees With Torn and Intact Anterior Cruciate Ligaments. Arthroscopy 2013;29:1954-62.

23. Comerford EJ, Tarlton JF, Avery NC, Bailey AJ, Innes JF. Distal femoral intercondylar notch dimensions and their relationship to composition and metabolism of the canine anterior cruciate ligament. Osteoarthritis Cartilage 2006;14:273-8.

24. Liu X, Ji G, Wang X, Kang H, Wang F. CT-based morphological analysis of the posterior femoral condyle in patients with trochlear dysplasia. Knee 2017;24:231-6.

25. Biedert RM, Bachmann M. Anterior-posterior trochlear measurements of normal and dysplastic trochlea by axial magnetic resonance imaging. Knee Surg Sports Traumatol Arthrosc 2009; 17:1225-30.

26. Bretin P, O'Loughlin PF, Suero EM, Kendoff D, Ostermeier S, Hüfner T, Krettek C, Citak M. Influence of femoral malrotation on knee joint alignment and intraarticular contract pressures. Arch Orthop Trauma Surg 2011;131:1115-20. 